\title{
Epidemiology of CTX-M-type extended-spectrum beta-lactamase (ESBL)-producing nosocomial - Escherichia coli infection in China
}

\author{
Huiqing Shi ${ }^{1,2 \dagger}$, Fengjun Sun ${ }^{1 \dagger}$, Jianhong Chen ${ }^{1}$, Qianyi Ou ${ }^{1}$, Wei Feng ${ }^{1}$, Xiaolan Yong ${ }^{2}$ and Peiyuan Xia ${ }^{1 *}$
}

\begin{abstract}
Background: Escherichia coli is one of the most common clinical pathogens causing nosocomial infection. The widespread cefotaxime-beta lactamases (CTX) has increased the multidrug resistance (MDR) of E. coli and has brought great trouble to the doctor treating the infection.

Methods: ESBL-positive E. coli isolates were collected from different hospitals in different areas and the minimal inhibitory concentration (MIC) was analyzed by the agar dilution method. The resistance gene types were detected using polymerase chain reaction (PCR) and the sequence types were determined by multilocus sequence typing (MLST).

Results: We found that the blaCTX-M-1 group and the blaCTX-M-9 group were the main CTX-M gene types, with many kinds of MLST gene types. Except for TEM with high isolate, SHV, OXA and VEB were relatively rare, while no PER and GES was detected. Most strains may have other resistance mechanisms, and the ESBL positive strains have high resistance not only to cephalosporins but also to other kinds of antibiotics.
\end{abstract}

Conclusion: The study provides wide epidemiological data and enables more effective infection control and treatment plans.

Keywords: Escherichia coli, Epidemiology, Extended-spectrum beta-lactamase, Multilocus sequence typing, Nosocomial infection

\section{Introduction}

Escherichia coli is one of the most common clinical pathogens causing nosocomial infection. For a long time, the widespread use of antibiotics to treat $E$. coli infectious disease has rapidly increased the multidrug resistance (MDR) of E. coli and hence has brought great trouble to the doctor treating the infection [1,2], especially with those strains producing extended-spectrum $\beta$-lactamase (ESBL). Although the Clinical and Laboratory Standards Institute (CLSI) reduced the necessity of ESBL screening and confirmatory tests and routine ESBL testing was no longer necessary in determining the dosage of antibiotics, ESBL testing is still useful for epidemiological purposes, because the ESBL-resistance gene carries a plasmid and transmits rapidly. After SHV-1 and TEM-1, the ESBL family of cefotaxime-beta lactamases (CTX) has been

\footnotetext{
* Correspondence: peiyuan_xia2013@163.com

${ }^{\dagger}$ Equal contributors

'Department of Pharmacy, Southwest Hospital, Third Military Medical University, Chongqing 400038, China

Full list of author information is available at the end of the article
}

reported in the literature to be increasing in frequency around the world $[3,4]$. CTX-M- $\beta$-lactamases can be divided into five groups according to their amino acid sequence identities. Different CTX genotypes have different hydrolysis reactions to $\beta$-lactam antibiotics $[5,6]$.

Obtaining accurate and prompt epidemiological data from CTX-M and other ESBL positive E. coli infections can enable an effective empirical therapy plan and infection control program. However, there has been little research in this area in China. In this study, we collected ESBLpositive E. coli from -different hospitals in different areas and analyzed the CTX-M and other ESBL gene type strains in China.

\section{Materials and methods \\ Bacterial strains}

A total of 342 isolated strains were collected from five general teaching hospitals in Chongqing, Henan, Tianjin, Hainan and Hebei from January 2012 to December 2013. Only samples collected from infected sites of inpatients of 
more than $48 \mathrm{~h}$ were included in the study. The sources of the clinical specimens were as follows: urine (107), sputum (65), blood (52), pus (34), abdominal fluid (22), bile (15), wound (12), skin (9), pleural fluid (6), vagina (5), joint (5), catheter (4), cerebrospinal fluid (3), drainage liquid (2) and paracentesis fluid (1). The isolated strains were tested using biochemical assays, the Vitek system (bioMe'rieux Vitek) and conventional biochemical and growth methods. The presence of ESBLs was evaluated in both the control strains and the recent clinical isolates. Double-disk diffusion was used to detect ESBL production. The cefotaxime (CTX) and ceftazidime (CAZ) disks in combination with clavulanate (CLA) were performed and interpreted by CLSI criteria for ESBL screening and disk confirmation tests [7]. Klebsiella pneumoniae ATCC 700603 and E. coli ATCC 25922 were used as positive and negative controls, respectively. The non-repeated ESBL positive $E$. coli were collected, and all the strains were collected from different patients.

\section{Antimicrobial susceptibility}

Antimicrobial susceptibility to a variety of drugs (including ampicillin, piperacillin, cefotaxime, cefepime, cefuroxime, cefoxitin, ceftazidime, aztreonam, imipenem, meropenem, ciprofloxacin, levofloxacin, gentamicin, amikacin, amoxicillin/clavulanic acid, ampicillin/sulbactam, piperacillin/tazobactam and trimethoprim-sulfamethoxazole) was evaluated by the agar dilution method according to CLSI guidelines (CLSI, 2014). E. coli ATCC 25922 and Pseudomonas aeruginosa ATCC 27853 were used as quality control strains.

\section{Molecular detection of ESBL}

All the isolates were screened by PCR using the specific primers for each couple primer in Table $1[8,9]$. The PCR products were sent for DNA sequencing and the sequence results were analyzed using the NCBI BLAST program (http://www.ncbi.nlm.nih.gov/).

\section{MLST analysis}

Multilocus sequence typing (MLST) was performed for the $E$. coli isolates [8]. The primers of seven housekeeping genes, including adk, fumC, gyrB, icd, mdh, purA and recA, were PCR-amplified, purified and sequenced. The sequences were then compared with the PubMLST database http://mlst.warwick.ac.uk/mlst/dbs/Ecoli), and each unique combination of alleles (the allelic profile) was designated as a sequence type (ST) [10].

\section{Statistical analysis}

The qualitative variables of the resistance rate to antimicrobial agents were compared using the chi-square test or the Fisher's exact test with SPSS 10.0 software package. $\mathrm{P} \leq 0.05$ was considered statistically significant.
Table 1 Primers used to amplify ESBL resistance genes

\begin{tabular}{|c|c|}
\hline Target(s) & Sequence \\
\hline \multirow[t]{2}{*}{ CTX-M group $I^{a}$} & GACGATGTCACTGGCTGAGC \\
\hline & AGCCGCCGACGCTAATACA \\
\hline \multirow[t]{2}{*}{ CTX-M group $\|^{b}$} & GCGACCTGGTTAACTACAATCC \\
\hline & CGGTAGTATTGCCCTTAAGCC \\
\hline \multirow[t]{2}{*}{ CTX-M group $\|^{c}$} & CGCTTTGCCATGTGCAGCACC \\
\hline & GCTCAGTACGATCGAGCC \\
\hline \multirow[t]{2}{*}{ CTX-M group IV } & GCTGGAGAAAAGCAGCGGAG \\
\hline & GTAAGCTGACGCAACGTCTG \\
\hline \multirow[t]{2}{*}{ CTX-M group $V^{e}$} & GCACGATGACATTCGGG \\
\hline & AACCCACGATGTGGGTAGC \\
\hline \multirow[t]{2}{*}{ TEM } & CATTTCCGTGTCGCCCTTATTC \\
\hline & CGTTCATCCATAGTTGCCTGAC \\
\hline \multirow[t]{2}{*}{ SHV } & AGCCGCTTGAGCAAATTAAAC \\
\hline & ATCCCGCAGATAAATCACCAC \\
\hline \multirow[t]{2}{*}{$O X A^{f}$} & GGCACCAGATTCAACTTTCAAG \\
\hline & GACCCCAAGTTTCCTGTAAGTG \\
\hline \multirow[t]{2}{*}{ VEB } & CATTTCCCGATGCAAAGCGT \\
\hline & CGAAGTTTCTTGGACTCTG \\
\hline \multirow[t]{2}{*}{ PER } & GCTCCGATAATGAAAGCGT \\
\hline & TTCGGCTTGACTCGGCTGA \\
\hline \multirow[t]{2}{*}{ GES } & AGTCGGCTAGACCGGAAAG \\
\hline & TTTGTCCGTGCTCAGGAT \\
\hline \multicolumn{2}{|c|}{ 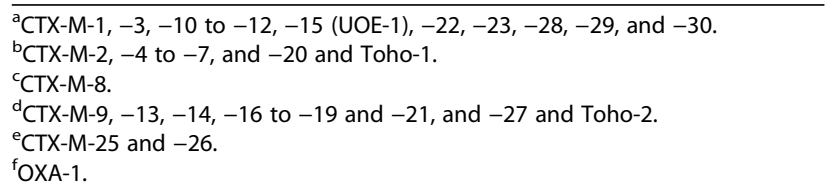 } \\
\hline
\end{tabular}

\section{Results}

During the study period, we obtained 130 ESBL-producing strains from clinical specimens. Among the strains, 21 were from Hebei, 19 from Hainan, 30 from Henan, 30 from Tianjin and 30 from Chongqing. The sources of the clinical specimens were as follows: - urine (36), blood (21), sputum (20), pus (18), abdominal fluid (10), bile (8), wound (7), skin (5), vagina (2), pleural fluid (2) and joint (1). The resistance rate of ESBL-producing $E$. coli from different areas is presented in Table 2. The ESBL positive strains were highly resistant to cephalosporins and fluoroquinolones. However, the strains responded better to the enzyme inhibitor and $\beta$-lactam antibiotics. Only three strains were resistant to imipenem, and two were resistant to meropenem. The cephalosporins and fluoroquinolones resistance rate of isolated strains from Hainan was lower than that of the strains from the other hospitals.

There were 126 (96.9\%) ESBL-producing isolates of amplified blaCTX-M genes, while only four strains were CTX-M negative. The positive rate of the ESBL resistance genes from different areas is presented in Table 3. The 
Table 2 Antimicrobial susceptibility of ESBL-producing Escherichia coli isolates from different areas

\begin{tabular}{|c|c|c|c|c|c|}
\hline Antimicrobial agent & Hainan $(n=19)$ & Hebei $(n=21)$ & Chongqing $(n=30)$ & Henan $(n=30)$ & Tianjin $(n=30)$ \\
\hline Ampicillin & 100 & 100 & 100 & 100 & 100 \\
\hline Piperacillin & 100 & 100 & 100 & 100 & 93.3 \\
\hline Cefotaxime & 94.7 & 100 & 100 & 100 & 90 \\
\hline Cefepime & 68.4 & $95.2^{*}$ & 93.3 & 90 & 83.3 \\
\hline Cefuroxime & 100 & 100 & 100 & 100 & 96.7 \\
\hline Cefoxitin & 15.8 & 23.8 & 36.7 & 16.7 & 20 \\
\hline Ceftazidime & 26.3 & $61.9^{*}$ & $56.7^{*}$ & $63.3^{*}$ & 53.3 \\
\hline Aztreonam & 5.3 & $61.9^{* *}$ & $73.3^{* *}$ & $76.7^{* *}$ & $60^{* *}$ \\
\hline Imipenem & 0 & 0 & 3.3 & 6.7 & 0 \\
\hline Meropenem & 0 & 0 & 6.7 & 0 & 0 \\
\hline Amoxicillin/Clavulanic acid & 84.2 & $9.5^{* *}$ & $43.3^{* *}$ & $50^{*}$ & $20^{* *}$ \\
\hline Ampicillin/Sulbactam & 73.7 & 71.4 & 80 & 83.3 & 76.7 \\
\hline Piperacillin/Tazobactam & 5.3 & 0 & 23.3 & 3.3 & 6.7 \\
\hline Trimethoprim-sulfamethoxazole & 78.9 & 85.7 & 83.3 & 83.3 & 73.3 \\
\hline Ciprofloxacin & 68.4 & 90.5 & 70 & 76.7 & 60 \\
\hline Levofloxacin & 47.4 & $90.5^{* *}$ & 70 & $76.7^{*}$ & 60 \\
\hline Amikacin & 5.3 & 0 & 16.7 & 6.7 & 6.7 \\
\hline Gentamycin & 52.6 & $85.7^{*}$ & 63.3 & 66.7 & 76.7 \\
\hline
\end{tabular}

**P $<0.01$ and ${ }^{*} \mathrm{P}<0.05$.

blaCTX-M-1 group and the blaCTX-M-9 group were detected at $66.9 \%(87 / 130)$ and $54.6 \%(71 / 130)$, respectively. No blaCTX-M-2, blaCTX-M-8, and blaCTX-M-25 groups were detected in the isolates. The blaCTX-M-9 group had CTX-M-65 and CTX-M-14. The blaCTX-M-1 group had CTX-M-3 and CTX-M-15. There were 110 (84.6\%) strains of amplified TEM. The OXA and SHV genes were detected at $21.5 \%(28 / 130)$ and $17.7 \%(23 / 130)$, respectively. Only 7 isolates were VEB positive. Neither PER nor GES was detected in the strains. Most strains had more than two resistance genes.

MLST results showed that 130 isolated strains had 42 gene types. The ST131 and ST167 were the most common genotypes with 19 and 12 strains separately. ST38, ST10, ST405 and ST2003 were less common with 9, 7, 7, and 6 isolates separately. For others, there were two genotypes having 5 strains, three having 4 strains, four having 3 strains, 9 having 2 strains and 18 having only 1 strain.

\section{Discussion}

This study presented the epidemiology of CTX-M E. coli from five hospitals in different cities of China. The results showed that the ESBL-producing isolates were highly resistant to both the cephalosporins and the fluoroquinolones confirming other reports $[8,11]$. This suggests that the ESBL resistance gene may exist with other resistance genes. Hence cephalosporin and fluoroquinolone are not considered to be effective choices for treatment of patients with ESBL-producing Enterobacteriaceae infection because of their relatively high resistance rates. However, the resistance rates of ceftazidime and aztreonam from Hainan hospital were lower than those from the other hospitals and other reports $[8,12,13]$. This demonstrates that other resistance mechanisms exist along with the ESBL in most strains. In our study, only four carbapenemresistant strains were detected, so carbapenems were recommended as reserve antimicrobials to treat ESBL bacterial

Table 3 Distribution of ESBL resistance genes in different areas

\begin{tabular}{llllll}
\hline & Hainan $(\mathbf{n}=\mathbf{1 9})$ & Hebei $(\mathbf{n}=\mathbf{2 1})$ & Chongqing $(\mathbf{n}=\mathbf{3 0})$ & Henan $(\mathbf{n}=\mathbf{3 0})$ & Tianjin $(\mathbf{n}=\mathbf{3 0})$ \\
\hline blaCTX-M-1 group & $57.9(11)$ & $42.9(9)$ & $83.3(25)$ & $83.3(25)$ & $56.7(17)$ \\
blaCTX-M-9 group & $68.4(13)$ & $71.4(15)$ & $53.3(16)$ & $26.7(8)$ & $63.3(19)$ \\
TEM & $63.2(12)$ & $85.7(18)$ & $96.7(29)$ & $83.3(25)$ & $86.7(26)$ \\
SHV & $0(0)$ & $14.3(3)$ & $30.0(9)$ & $13.3(4)$ & $23.3(7)$ \\
OXA & $10.5(2)$ & $33.3(7)$ & $26.7(8)$ & $16.7(5)$ & $20.0(6)$ \\
VEB & $0(0)$ & $0(0)$ & $16.7(5)$ & $0(0)$ & $6.7(2)$ \\
\hline
\end{tabular}


infections. Though the five hospitals were widely separated in China, the resistance rate of clinical isolates from the hospitals to the tested drugs was similar except the strains from Hainan, which showed that the ESBL-producing strains had a similar drug resistance spectrum.

All ESBL-positive strains from the five hospitals were analyzed for blaCTX-M genes by PCR assay. The results showed that almost all isolates (126/130) - carried blaCTXM. Obviously, the blaCTX-M type is the most common ESBL resistance gene in China, as most reports indicate. The CTX-M ESBL E. coli positive rate is higher than that reported abroad $[8,14,15]$, which is due to the wide use of $\beta$-lactam antibiotics. At the same time, the main CTX-M gene types of the five cities were CTX-M-14 and CTX-M15 , which is confirmed by most research in China $[8,16]$. At the same time, the positive rates of the blaCTX-M-1 group and the blaCTX-M-9 group have some differences, which suggest that there are regional epidemiological characteristics. This result suggests that CTX-M-14 is widely distributed among hospital infection patients, and that when doctors doubt whether ESBL E. coli infection is present, they try to avoid using the $\beta$-lactam antibiotics. The $\beta$-lactam/ $\beta$-lactamase inhibitor combinations, carbapenems and amikacin should first be considered. The results for the TEM, SHV, OXA, VEB, PER and GES genes assay showed that TEM-positive $E$ coli strains were also very common in the five hospitals, while the others were relatively rare, which was consistent with the results in previous studies $[17,18]$. At the same time, the positive rates of the TEM, SHV, OXA and VEB gene have some differences among areas, indicating that there are some regional epidemiological characteristics. The study showed that the CTX-M-type ESBL was the most common resistance gene type. As the different ESBL resistance genes have different hydrolysis capabilities with different $\beta$-lactams antibacterials, so the different ESBL distributions should be considered in antibacterial use.

All the clinically isolated strains belonged to 42 gene types. ST131 was the most common with 19 strains, which was confirmed by domestic and international research [8,19-21]. ST167 was the second with 12 strains. ST167 was also a common genotype in $E$ coli in foreign research [22], which was seldom reported in China [23]. ST167 was listed a lineage with a potential extended host spectrum genotype. Except for ST131 and ST167, no other types had more than 10 strains. There was no obvious relationship between the MLST gene type and ESBL resistance gene type. This result suggests that the ESBL gene is widely distributed in different MLST gene types, which is confirmed by other reports.

This research has two limitations: first, the clinical information was not acquired, and we could not further analyze the risk factors for ESBL E. coli infections; second, the number of strains was not large enough to display epidemiological features. Generally speaking, the study examined the drug resistance and genotypic epidemiology of CTX-M type clinically isolated E. coli. We found that the CTX-M is still the primary genotype of ESBL in China, and that the ESBL positive strains have higher resistance. The CTX-M-14 and CTX-M-15 are the most common resistance gene types and ST131 is the predominant clonal group. This study will provide reference data to enable relevant infection control and treatment.

\section{Competing interests}

The authors declare that they have no competing interests.

\section{Authors' contributions}

HS and FS carried out the protocol design and drafted this manuscript. JC carried out the collection of data, the analysis and interpretation of these data. QO and WF carried out the collection and analysis of data for this study. XY participated in the collection of data for this study. PX carried out the protocol design, the analysis and interpretation of these data, and drafted and revised the content of this manuscript. All authors read and approved the final manuscript.

\section{Acknowledgements}

This study was supported by the National Natural Science Foundation of China (No. 81373451).

\section{Author details}

${ }^{1}$ Department of Pharmacy, Southwest Hospital, Third Military Medical University, Chongqing 400038, China. '2Department of Clinical Pharmacy, General Hospital of Chengdu Military Region, Chengdu, Sichuan Province 610083, China.

Received: 8 October 2014 Accepted: 8 January 2015

Published online: 16 January 2015

\section{References}

1. Trecarichi EM, Cauda R, Tumbarello M. Detecting risk and predicting patient mortality in patients with extended-spectrum beta-lactamase-producing Enterobacteriaceae bloodstream infections. Future Microbiol. 2012;7:1173-89.

2. Kanwar N, Scott HM, Norby B, Loneragan GH, Vinasco J, McGowan M, et al. Effects of ceftiofur and chlortetracycline treatment strategies on antimicrobial susceptibility and on tet(A), tet(B), and bla CMY-2 resistance genes among $E$. coli isolated from the feces of feedlot cattle. PLoS One. 2013;8:e80575

3. Woerther PL, Burdet C, Chachaty E, Andremont A. Trends in human fecal carriage of extended-spectrum beta-lactamases in the community: toward the globalization of CTX-M. Clin Microbiol Rev. 2013;26:744-58.

4. D'Andrea MM, Arena F, Pallecchi L, Rossolini GM. CTX-M-type beta-lactamases: a successful story of antibiotic resistance. Int J Med Microbiol. 2013;303:05-17.

5. Pitout JD, Hossain A, Hanson ND. Phenotypic and molecular detection of CTX-M-beta-lactamases produced by Escherichia coli and Klebsiella spp. J Clin Microbiol. 2004;42:5715-21

6. Bonnet R. Growing group of extended-spectrum beta-lactamases: the CTX-M enzymes. Antimicrob Agents Chemother. 2004;48:1-14.

7. Clinical Laboratory Standards Institute. Performance standards for antimicrobial susceptibility testing; 24th informational supplement. CLSI document M100-S24. Wayne, PA: Clinical Laboratory Standards Institute; 2014. p. 110-2.

8. Xia S, Fan X, Huang Z, Xia L, Xiao M, Chen R, et al. Dominance of CTX-M-type extended-spectrum beta-lactamase (ESBL)-producing Escherichia coli isolated from patients with community-onset and hospital-onset infection in China. PLoS One. 2014;9:e100707.

9. Dallenne C, Da Costa A, Decré D, Favier C, Arlet G. Development of a set of multiplex PCR assays for the detection of genes encoding important betalactamases in Enterobacteriaceae. J Antimicrob Chemother. 2010;65:490-5.

10. Park SH, Byun JH, Choi SM, Lee DG, Kim SH, Kwon JC, et al. Molecular epidemiology of extended-spectrum beta-lactamase-producing Escherichia coli in the community and hospital in Korea: emergence of ST131 producing CTX-M-15. BMC Infect Dis. 2012;12:149. 
11. Tóth $A$, Kocsis B, Damjanova I, Kristóf $K$, Jánvári L, Pászti J, et al. Fitness cost associated with resistance to fluoroquinolones is diverse across clones of Klebsiella pneumoniae and may select for CTX-M-15 type extended-spectrum beta-lactamase. Eur J Clin Microbiol Infect Dis. 2014;33:837-43.

12. Hayakawa K, Gattu S, Marchaim D, Bhargava A, Palla M, Alshabani K, et al. Epidemiology and risk factors for isolation of Escherichia coli producing CTX-M-type extended-spectrum beta-lactamase in a large U.S. Medical Center. Antimicrob Agents Chemother. 2013;57:4010-8.

13. Ripoll A, Galán JC, Rodríguez C, Tormo N, Gimeno C, Baquero F, et al. Detection of resistance to beta-lactamase inhibitors in strains with CTX-M beta-lactamases: a multicenter external proficiency study using a welldefined collection of Escherichia coli strains. J Clin Microbiol. 2014;52:122-9.

14. Eller C, Leistner R, Guerra B, Fischer J, Wendt C, Rabsch W, et al. Emergence of extended-spectrum beta-lactamase (ESBL) CTX-M-8 in Germany. J Antimicrob Chemother. 2014;69:562-4.

15. Carvalho-Assef AP, Pereira PS, Albano RM, Berião GC, Tavares CP, Chagas TP, et al. Detection of NDM-1-, CTX-M-15-, and qnrB4-producing Enterobacter hormaechei isolates in Brazil. Antimicrob Agents Chemother. 2014;58:2475-6.

16. Zhao WD, Yan P, Guan HN, Zhang QZ. Characterization of CTX-M-type extended-spectrum beta-lactamase in clinical clones of Escherichia coli in Southwest China. J Basic Microbiol. 2014;54:247-52.

17. Zhang Z, Guo X, Zhang Q. Prevalence characterization of extended-spectrum $\beta$-lactamases among Escherichia coli isolates collected in Zhengzhou. J Clin Lab Anal. 2009;23:404-7.

18. Zhang C, Xu X, Pu S, Huang S, Sun J, Yang S, et al. Characterization of carbapenemases, extended spectrum $\beta$-lactamases, quinolone resistance and aminoglycoside resistance determinants in carbapenem-nonsusceptible Escherichia coli from a teaching hospital in Chongqing, Southwest China. Infect Genet Evol. 2014;27:271-6.

19. Zhang L, Lü X, Zong Z. The emergence of blaCTX-M-15-carrying Escherichia coli of ST131 and new sequence types in Western China. Ann Clin Microbiol Antimicrob. 2013;12:35.

20. Al-Agamy MH, Shibl AM, Hafez MM, Al-Ahdal MN, Memish ZA, Khubnani H. Molecular characteristics of extended-spectrum beta-lactamase-producing Escherichia coli in Riyadh: emergence of CTX-M-15-producing E. coli ST131. Ann Clin Microbiol Antimicrob. 2014;13:4.

21. Nicolas-Chanoine MH, Robert J, Vigan M, Laouénan C, Brisse S, Mentré F, et al. Different factors associated with CTX-M-producing ST131 and non-ST131 Escherichia coli clinical isolates. PLoS One. 2013;8:e72191.

22. Schink AK, Kadlec K, Kaspar H, Mankertz J, Schwarz S. Analysis of extendedspectrum- $\beta$-lactamase-producing Escherichia coli isolates collected in the GERM-Vet monitoring programme. J Antimicrob Chemother. 2013;68:1741-9.

23. Zhang $X$, Lou D, Xu Y, Shang $Y$, Li D, Huang $X$, et al. First identification of coexistence of blaNDM-1 and blaCMY-42 among Escherichia coli ST167 clinical isolates. BMC Microbiol. 2013;13:282.

\section{Submit your next manuscript to BioMed Central and take full advantage of:}

- Convenient online submission

- Thorough peer review

- No space constraints or color figure charges

- Immediate publication on acceptance

- Inclusion in PubMed, CAS, Scopus and Google Scholar

- Research which is freely available for redistribution

Submit your manuscript at www.biomedcentral.com/submit 